Rev. Int. Contam. Ambie. 37, 27-38, 2021

https://doi.org/10.20937/RICA.53632

\title{
ESTIMACIÓN DEL BIOGÁS GENERADO EN SITIOS DE DISPOSICIÓN FINAL DEL ESTADO DE MÉXICO
}

Estimation of the biogas generated in final disposal sites in the State of Mexico

\section{Paulina R. CÁRDENAS-MORENO, Ana Belem PIÑA-GUZMÁN y Fabián ROBLES-MARTÍNEZ*}

Instituto Politécnico Nacional, Unidad Profesional Interdisciplinaria de Biotecnología, Av. Acueducto s/n, Barrio La Laguna, Col. Ticomán, 07340 Ciudad de México, México.

*Autor para correspondencia: froblesm@ipn.mx

(Recibido: mayo de 2019; aceptado: junio de 2020)

Palabras clave: emisiones, metano, rellenos sanitarios.

\section{RESUMEN}

En México se desconoce la información sobre el manejo de la mayoría de los sitios de disposición final (SDF), al igual que las cantidades de biogás producidas por la degradación natural de materia orgánica contenida en los residuos sólidos urbanos eliminados. El biogás está compuesto principalmente por metano y dióxido de carbono, ambos considerados gases de efecto invernadero (GEI). Existen varios modelos para el cálculo de emisiones de GEI provenientes de SDF, entre los que encuentran el Modelo Mexicano de Biogás 2.0 (MMB) y el modelo del Grupo Intergubernamental de Expertos sobre el Cambio Climático. El objetivo del presente trabajo fue estimar la cantidad de biogás generada en SDF del estado de México (EM) utilizando ambos modelos, con el objetivo de proyectar la generación para un periodo de 50 años desde la apertura de los sitios. Con este fin se identificaron $30 \mathrm{SDF}$ del EM en un radio de $50 \mathrm{~km}$ desde el centro de la ciudad de México y se recabó información mediante visitas y entrevistas para alimentar la hoja de cálculo de los modelos. Se calculó la generación anual de biogás para 15 rellenos sanitarios, seis sitios controlados y nueve sitios no controlados (clasificados de acuerdo con la Norma Oficial Mexicana NOM-083-SEMARNAT-2003). Se estimó una generación de biogás para los sitios de estudio de 198 kt de $\mathrm{CH}_{4}$ para 2017, $193 \mathrm{kt}$ para 2020, $85 \mathrm{kt}$ para 2030 y $12 \mathrm{kt}$ de $\mathrm{CH}_{4}$ para 2050 con el MMB. Los resultados se utilizaron para evaluar su posible impacto en el ambiente de la Ciudad de México y municipios contiguos.

Key words: emissions, methane, landfill.

\begin{abstract}
Currently, the specific information on the management of most of the final disposal sites (FDS) in Mexico is unknown, as well as the volumes of biogas produced by the natural degradation of the organic matter contained in the disposed municipal solid waste (MSW). Biogas is mainly composed of methane and carbon dioxide, both considered greenhouse gases (GHGs). Several models have been developed for the calculation of GHG emissions from FDS, among which the Mexican Biogas Model 2.0 (MBM) and the Intergovernmental Panel on Climate Change model can be found. The aim of the
\end{abstract}


present work was to estimate the amount of biogas generated in FDS of the State of Mexico by using the aforementioned models, to project the generation of biogas for a period of 50 years since the opening of the sites. To this aim, 30 FDS located in the $\mathrm{SM}$ comprised in a $50 \mathrm{~km}$ radius from the center of Mexico City were identified and data collected through visits and interviews were entered into the model's spreadsheet. The annual generation of biogas was estimated for 15 landfills, six controlled sites and nine uncontrolled sites, classified according to the Official Mexican Standard NOM083-SEMARNAT-2003. Biogas generation was calculated for the studied sites as $198 \mathrm{kt} \mathrm{CH}_{4}$ in 2017, $193 \mathrm{kt} \mathrm{CH}_{4}$ in 2020, $85 \mathrm{kt} \mathrm{CH}_{4}$ in 2030, and $12 \mathrm{kt} \mathrm{CH}_{4}$ for 2050. The results were used to evaluate their possible environmental impact in Mexico City and the adjacent municipalities.

\section{INTRODUCCIÓN}

El tratamiento principal de los residuos sólidos urbanos (RSU), en los países en vías de desarrollo consiste en enterrarlos en los sitios de disposición final (SDF), los cuales son considerados como el método más adecuado y económico de eliminación de residuos (Friedrich y Trois 2011). En México se reporta que el $78.54 \%$ de los residuos generados se entierran en SDF (SEMARNAT 2019), mientras que en Estados Unidos el 52.6 \% de los RSU fueron depositados en rellenos sanitarios en año 2014 (Lee et al. 2017).

En México, dichos SDF incluyen a los rellenos sanitarios (RESA), pero también a los sitios controlados (SC) y los tiraderos a cielo abierto o sitios no controlados (SNC). La Norma Oficial Mexicana NOM-083-SEMARNAT-2003 (SEMARNAT 2003) rige la apertura, selección, diseño, construcción, operación, monitoreo, clausura y obras complementarias de RESA. Asimismo, el manejo de los RSU está a cargo del gobierno municipal (tercero en los tres niveles de gobierno), es decir, cada municipio debe hacerse cargo de los residuos generados por su población. El Estado de México (EM), la entidad federativa más poblada de la República Mexicana (16 225409 habitantes en 2015 [INEGI 2019]), se divide en 125 municipios; además, limita con la Ciudad de México (CdMx) (8 985339 habitantes [INEGI 2019]), y juntos constituyen el área metropolitana más grande del país y parte de la zona metropolitana del valle de México (ZMVM) (Fig. 1). Por su alta densidad poblacional (proporcional a la generación de RSU), en el EM se recolectan aproximadamente $13 \mathrm{kt} / \mathrm{día}$ de RSU (INEGI 2015) para ser valorizadas y, en su mayoría, depositadas en los SDF dicha entidad, donde de acuerdo con trabajos previos (González-Cruz 2013, Cárdenas-Moreno 2014, Cárdenas-Moreno et al. 2019) se han observado graves deficiencias en la gestión de RSU. Esta problemática creció debido a que, en diciembre del 2011, el único RESA de la CdMx (Bordo Poniente), que recibía la mayor parte de los RSU de esta ciudad, fue cerrado después de 25 años de operación. En consecuencia, cuatro SDF del EM (La Cañada, El Milagro, municipio de Ixtapaluca, Cuautitlán y Chicoloapan) comenzaron a recibir $7.35 \mathrm{kt} /$ día de RSU procedentes de la CdMx, aumentando de manera considerable los volúmenes de RSU normalmente dispuestos en estos sitios (SEDEMA 2015).

En los SDF se lleva a cabo la degradación de materia orgánica de los RSU. En condiciones de anaerobiosis, se inicia una serie de procesos fisicoquímicos y biológicos que, dependiendo de la composición de los residuos y de las condiciones ambientales, determinarán la cantidad y composición de los lixiviados y del biogás. Numerosos estudios enumeran diversos componentes encontrados en el biogás producido en los RESA, siendo el $\mathrm{CH}_{4}$ y el $\mathrm{CO}_{2}$ los componentes principales (Themelis y Ulloa 2007, Chiemchaisri y Visvanathan 2008). Como ejemplo, en el Cuadro I se muestra la composición típica del biogás de un RESA, cuyos componentes incluyen algunos compuestos orgánicos volátiles no metánicos (COVNM) o precursores de ozono troposférico.

En México, donde gran parte de los SDF de RSU no cuentan con sistemas de recuperación de biogás, la emisión de éste podría constituir un problema grave por el impacto ambiental causado, por lo que es necesario al menos estimar los volúmenes generados.

El cálculo de emisiones de gases de efecto invernadero (GEI) se lleva acabo para conocer la magnitud del impacto de una actividad determinada sobre el cambio climático, en este caso la operación de SDF de RSU. A este respecto, el INECC (2018) reporta que en México el sector residuos aporta el $6.56 \%$ del total de los GEI emitidos. El biogás resultante de la descomposición de los residuos en SDF se puede calcular con ayuda de modelos que resumen la química muy compleja que ocurre durante la descomposición 


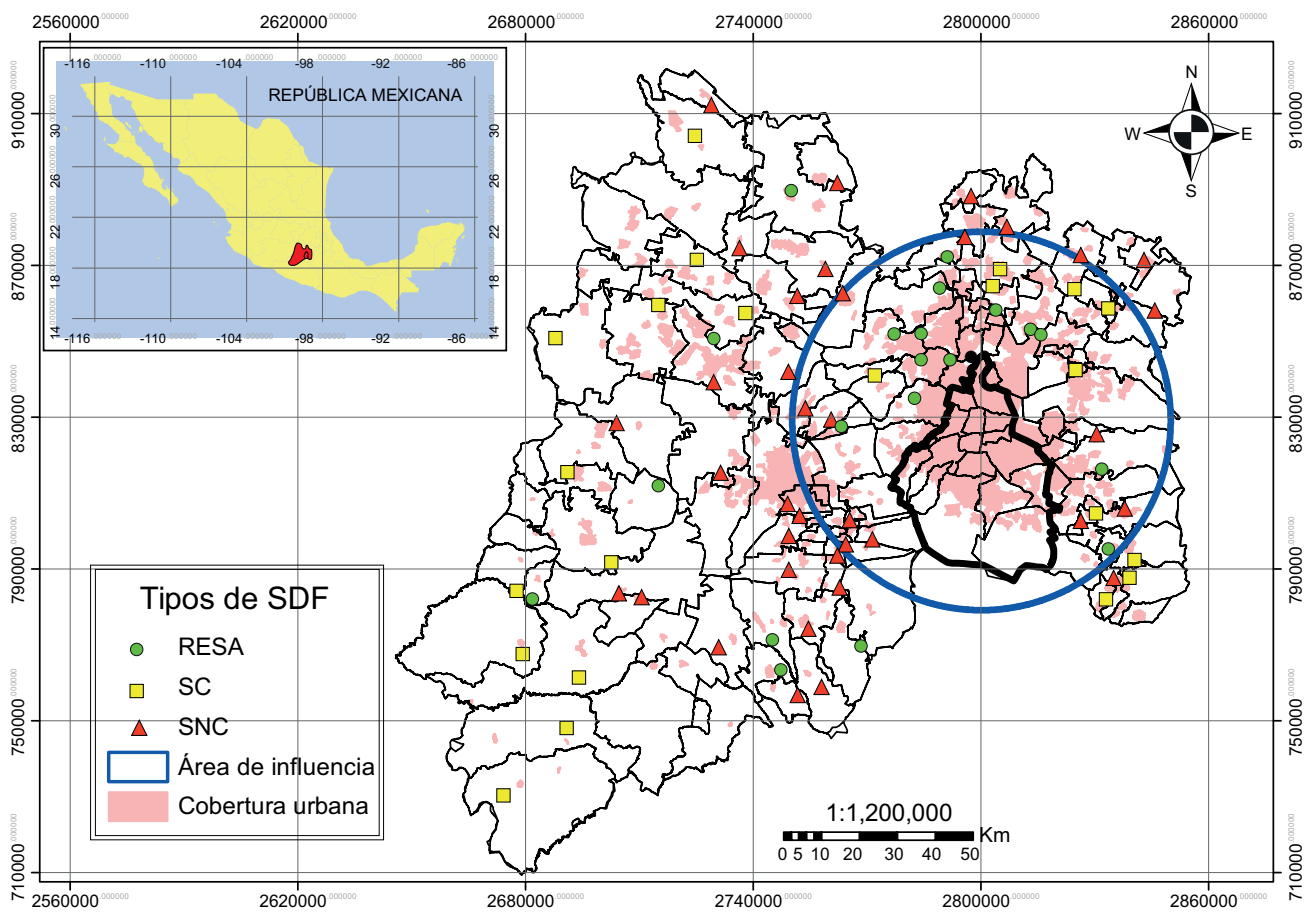

Fig. 1. Tipos de SDF en el estado de México y en el área de estudio en 2012.

CUADRO I. COMPOSICIÓN TÍPICA DEL BIOGÁS PRODUCIDO EN SDF.

\begin{tabular}{lc}
\hline Componente & \% en volumen \\
\hline Metano $\left(\mathrm{CH}_{4}\right)$ & $50-60$ \\
Dióxido de carbono $\left(\mathrm{CO}_{2}\right)$ & $40-50$ \\
Nitrógeno $\left(\mathrm{N}_{2}\right)$ & $2-3$ \\
Sulfuro de hidrógeno $\left(\mathrm{H}_{2} \mathrm{~S}\right)$ & $1-2$ \\
Hidrógeno $\left(\mathrm{H}_{2}\right)$ & $<1$ \\
Oxígeno $\left(\mathrm{O}_{2}\right)$ & $<1$ \\
Monóxido de carbono $(\mathrm{CO})$ & Traza \\
Amoniaco $\left(\mathrm{NH}_{2}\right)$ & Traza \\
Hidrocarburos aromáticos y cíclicos & Traza \\
Compuestos orgánicos volátiles & Traza \\
\hline
\end{tabular}

Elaboración propia con base en Themelis y Ulloa (2007) y Chiemchaisri y Visvanathan (2008).

biológica de RSU. Existen diferentes entidades que han elaborado metodologías para el cálculo de emisiones de GEI provenientes de actividades específicas. El Modelo Mexicano de Biogás 2.0 (MMB) (US-EPA y SCS 2009) provee una herramienta de estimación automatizada para cuantificar la generación y recuperación de biogás en SDF de México. De acuerdo con Colomer-Mendoza et al. (2016), quienes estimaron las emisiones de biogás de un relleno sanitario en el estado de Jalisco (México) utilizando varios modelos, el MMB se ajusta de manera adecuada a la generación real de biogás. También Urrego-Martínez y Rodríguez-Miranda (2016) utilizaron varios modelos para estimar el biogás generado en el relleno sanitario de Doña Juana en Colombia, encontrando que el MMB presenta el mejor resultado de estimación promedio de biogás y ajuste estadístico adecuado para este caso de estudio.

Por otro lado, el modelo del Grupo Intergubernamental de Expertos sobre el Cambio Climático (IPCC, por sus siglas en inglés) es uno de los más utilizados en el mundo para el cálculo de emisiones de metano (Ngnikam et al. 2002, Kumar et al. 2004, Chiemchaisri y Visvanathan 2008, Machado et al. 2009, Mou et al. 2015), ya que establece metodologías para países, sitios regionales y municipales. De este modo, conociendo la situación actual del manejo y disposición de RSU en el área de estudio, se aplicaron tanto el modelo del IPCC como el MMB para estimar la cantidad de biogás generado en SDF del Estado de México con el objetivo de inferir la posible afectación al ambiente, a partir de datos de generación de RSU de 2012 y el tipo de disposición final. Además, se estimó la generación de biogás para un periodo de 50 años a partir de la apertura de los SDF. 


\section{MATERIALES Y MÉTODOS}

Se realizó una búsqueda integral de información (tanto bibliográfica como mediante levantamiento de encuestas, descrita en el siguiente apartado) acerca de la generación de RSU y su disposición en los SDF del EM, para caracterizar dichos sitios y alimentar los modelos de estimación de generación de biogás.

\section{Búsqueda de información}

Primeramente, se delimitó el área de estudio, para lo cual se trazó un círculo de $50 \mathrm{~km}$ de radio desde el centro de la CdMx hacia el EM, y dentro de ésta se localizaron todos los SDF cuyas emanaciones de biogás pudieran tener un impacto negativo en el ambiente de la CdMx (Fig. 1). Posteriormente se realizó una búsqueda tanto bibliográfica (consultando sitios de internet correspondientes a instituciones gubernamentales) como en bases de datos del Instituto Nacional de Estadística y Geografía (INEGI), la Secretaría de Medio Ambiente y Recursos Naturales (SEMARNAT) y la Secretaría de Medio Ambiente del Estado de México (SMA). Asimismo, se tomó en cuenta la información de campo obtenida por González-Cruz (2013) mediante la aplicación de cuestionarios a los encargados de los SDF del EM sobre la problemática actual en los sitios y los métodos de operación en cada uno de ellos. Los sitios estudiados se listan en el cuadro II. La información obtenida de las encuestas fue analizada y comparada con las bases de datos oficiales, cuyos datos fueron reportados por González-Cruz (2013), quien obtuvo información sobre fechas de apertura y clausura de los SDF, ubicación geográfica, tipo de manejo y cantidades recibidas de RSU. Dicha información se utilizó para alimentar las hojas de cálculo del MMB y del IPCC, además de que se analizó en un sistema de información geográfica.

\section{Clasificación de los SDF}

Los SDF del área de estudio se categorizaron de acuerdo con la NOM-083-SEMARNAT-2003, que los clasifica en 1) rellenos sanitarios (RESA), 2) sitios controlados (SC) y 3) sitios no controlados (SNC), con base en las características de su operación y el tonelaje de RSU que los RESA reciben diariamente (A1: más de 750 t/día; A2: entre 750 y 100 t/día; B: entre 100 y 50 t/día; C: entre 50 y 10 t/día, y D: menos de 10 t/día).

\section{Aplicación del MMB}

El MMB dispone de cinco hojas de cálculo del programa Excel. Las tres primeras deben ser llenadas manualmente con los datos particulares de cada SDF (año de apertura y clausura, cantidad anual de residuos dispuestos, tipo de manejo, fracción orgánica e inorgánica, e incremento anual del $1 \%$ en la disposición de residuos con base en el aumento poblacional [CONAPO, 2019]). En las dos últimas hojas se obtienen de manera automática los resultados estimados de las emisiones, es decir, las tabulaciones y las proyecciones de generación de biogás para un SDF a lo largo de un periodo de tiempo. Este modelo realiza una proyección para 50 años posteriores al año de apertura de cada sitio.

Dentro del registro de datos para cada sitio, la hoja de cálculo permite diferenciar entre el tipo de sitio a evaluar, es decir su clasificación de acuerdo con la NOM-0.83-SEMARNAT-2003 (dada por el

CUADRO II. LISTA Y CLASIFICACIÓN DE LOS SDF QUE SE ENCONTRARON DENTRO DEL RADIO DE ESTUDIO.

\begin{tabular}{llllll}
\hline Municipio & Tipo & Municipio & Tipo & Municipio & Tipo \\
\hline Amecameca & SNC & Otumba & SNC & Isidro Fabela & SC \\
Ayapango y Juchitepec & RESA & San Martin de las Pirámides & SNC & Naucalpan de Juárez & RESA \\
Cocotitlán & SC & Tecámac & RESA & Nicolás Romero & RESA \\
Chicoloapan & SNC & Temascalapa & SNC & Papalotla & SC \\
Ixtapaluca & RESA & San Juan Teotihuacán & SC & Atizapán de Zaragoza & RESA \\
Cuautitlán Izcalli & RESA & Capulhuac & SNC & Tlalnepantla de Baz & RESA \\
Tepotzotlán & RESA & Otzolotepec* & SC & Tultepec & RESA \\
Villa del Carbón & SNC & Tianguistenco* & SNC & Tultitlán* & RESA \\
Huehuetoca & RESA & Xalatlaco & SNC & Zumpango & SC \\
Ecatepec & RESA & Xonacatlán & RESA & Bordo Poniente* & RESA \\
\hline
\end{tabular}

SNC: sitio no controlado, RESA: relleno sanitario, SC: sitio controlado.

*Clausurado. 
tipo manejo y operación, así como infraestructura). De igual manera, si no hay datos específicos del sitio, se pueden utilizar los valores por defecto que proporciona el modelo, los cuales son asignados de acuerdo con la región en que se localice. En el presente estudio se utilizaron los datos proporcionados por los encargados de los sitios, reportados en González-Cruz (2013) y por medio de la entrevista al encargado del SDF de Tecámac (Zúñiga 2014).

El MMB emplea la siguiente ecuación de degradación de primer grado:

$Q_{L F G}=\sum_{t=1}^{n} \sum_{j=0.1}^{1} 2 k L_{0}\left[\frac{M i}{10}\right]\left(e^{-k t_{i j}}\right)(M C F)(F)$

donde $Q_{\mathrm{LFG}}$ es el flujo de biogás máximo esperado $\left[\mathrm{m}^{3} /\right.$ año], $i$ es el incremento en tiempo de 1 año, $n$ es el año del cálculo menos el año inicial de disposición de residuos, $j$ es el incremento de tiempo en 0.1 años, $k$ es el índice de generación de $\mathrm{CH}_{4}$ (1/año), $L_{0}$ es la generación potencial de $\mathrm{CH}_{4}\left(\left[\mathrm{~m}^{3} / \mathrm{t}\right), M i\right.$ es la masa de residuos dispuestos en el año $i(\mathrm{t}), t_{\mathrm{ij}}$ es la edad de la sección $j$ de la masa de residuos $M i$ dispuestos en el año $i$ (años decimales), $M C F$ es el factor de corrección para el $\mathrm{CH}_{4}$ y $F$ es el factor de ajuste por incendios.

\section{Aplicación del Modelo del IPCC}

Este modelo consta de dos metodologías, la primera para residuos separados por fracción degradable (varias fases) y la segunda para residuos mezclados. Se eligió la segunda alternativa para residuos mezclados (ecuación 2), que es un modelo de fase única basado en los residuos brutos, debido a que en ningún sitio se contaba con un estudio de caracterización por tipo de residuos. La metodología completa puede consultarse en las directrices del IPCC para inventarios nacionales de GEI (Pipatti y Svardal 2007).

$\mathrm{CH}_{\text {generado }_{T}}=\mathrm{DDOCm}_{\text {descomp }_{T}} F\left(\frac{16}{12}\right)$

donde $\mathrm{CH}_{4 \text { generado }}$ es la cantidad de $\mathrm{CH}_{4}$ generado $\left(\mathrm{m}^{3} / \mathrm{año}\right)$ a partir del material en descomposición del año estudiado (t), DDOC $C_{m \text { descomp }}$ es el $\mathrm{CH}_{4}$ descompuesto durante el año estudiado (t), $F$ es la fracción volumétrica de $\mathrm{CH}_{4}$ en el gas de vertedero generado (0.5), 16/12 es el cociente de pesos moleculares $\mathrm{CH}_{4} / \mathrm{C}$. Esta metodología demanda los mismos requerimientos específicos de los sitios que el MMB. Para el manejo de las ecuaciones nuevamente se utilizaron hojas de Excel.

\section{Validación del MMB}

Para validar un modelo de proyección de emisiones se requieren hacer mediciones en el sitio; sin embargo, no todo el biogás emitido puede capturarse, ya que no es un sistema completamente cerrado. En ese sentido, el volumen de biogás que se recupera mediante un sistema de ductos si es conocido. En este caso, sólo el SDF de Tecámac contaba con dicha información para probar si el modelo se ajustaba a datos medidos. El MMB proporciona también la curva de recuperación estimada (RE) de biogás, si se le suministran los datos requeridos, por lo que se realizó la comparación de los datos estimados con los datos medidos el RESA del SDF Tecámac. Dichos datos consistían en los resultados de la medición del flujo de biogás de cinco años. Además, se obtuvo información sobre el número de jornadas por año en que estuvo sin operar el sistema de recuperación de biogás.

\section{RESULTADOS Y DISCUSIÓN}

\section{Obtención de información y clasificación de los SDF}

Se obtuvo información para la estimación de generación de biogás en 30 municipios del Estado de México, todos comprendidos en el área de estudio. De los SDF estudiados, 15 (50\%) operaban como RESA, seis (20\%) como SC y nueve (30\%) como SNC. Cabe mencionar que cuatro (13\%) se encontraban ya clausurados al momento de aplicar la encuesta, entre ellos el RESA Bordo Poniente (RESA-BP). Además, se estimó que, para finales de 2011, en los SDF estudiados se depositaban diariamente alrededor de 10.84 kt de RSU, además de los residuos dispuestos en el RESA-BP (alrededor de $7.55 \mathrm{kt} /$ día de RSU), para una disposición total de $18.4 \mathrm{kt} /$ día RSU (alrededor de 6714.18 kt/año). Se encontró que los 15 RESA estudiados (ubicados en los municipios aledaños a la CdMx y también con la mayor población del EM), recibieron más de 7 millones de toneladas en el año de estudio, es decir más del $97 \%$ de lo dispuesto en el área de estudio. La información particular de los sitios de estudio se encuentra disponible en Cárdenas-Moreno (2014).

\section{Estimación del biogás generado utilizando los modelos MMB y del IPCC}

En el cuadro III se muestra la cantidad depositada de RSU por tipo de SDF en 2012 y la proyección de la cantidad estimada de biogás que se generará para los años posteriores empleando el MMB. Debido a que la generación de biogás es directamente proporcional a 
CUADRO III. PROYECCIÓN DE LA GENERACIÓN ESTIMADA DE BIOGÁS POR TIPO DE SDF EMPLEANDO EL MMB.

\begin{tabular}{|c|c|c|c|c|c|c|}
\hline \multirow[t]{2}{*}{ Tipo de SDF } & \multicolumn{2}{|c|}{ Disposición final (t RSU/año) (\%) } & \multicolumn{4}{|c|}{ Generación de biogás $^{1}(\mathrm{t} / \mathrm{año})^{2}$} \\
\hline & \multicolumn{2}{|c|}{2012} & 2017 & 2020 & 2030 & 2050 \\
\hline $\operatorname{RESA}(15 / 30)$ & 3962473.065 & 97 & 192505.44 & 188052.41 & 83866.41 & 11555.32 \\
\hline $\mathrm{SC}(6 / 30)$ & 82161.5 & 1 & 769.75 & 534.50 & 128.66 & 40.30 \\
\hline SNC $(9 / 30)$ & 157315.0 & 2 & 4455.66 & 3902.59 & 1340.27 & 278.77 \\
\hline Total de $30 \mathrm{SDF}$ & 4201949.57 & 100 & 197730.85 & 192489.50 & 85335.34 & 11874.39 \\
\hline
\end{tabular}

SDF: sitio de disposición final, MMB: Modelo Mexicano de Biogás, SNC: sitio no controlado, RESA: relleno sanitario, SC: sitio controlado.

${ }^{1}$ Incluye la estimación de los sitios clausurados, ${ }^{2}$ se realizó la conversión de unidades a t/año utilizando la densidad del $\mathrm{CH}_{4}$ $\left(25^{\circ} \mathrm{C}, 1 \mathrm{~atm}\right)$.

la cantidad de RSU dispuesta en los sitios, se observa que los RESA estudiados son los sitios que estarán produciendo la mayor cantidad de biogás.

Por lo anterior se puede afirmar que es necesario llevar un control adecuado de la emisión de biogás en un RESA, además de contar con la tecnología para su recuperación y valorización. Por ejemplo, que el biogás sea conducido mediante una red superficial de tuberías y se aproveche para la producción de electricidad por medio de motores de combustión interna y turbinas. Sin embargo, la NOM-083-SEMARNAT-2003 establece que la quema del gas emitido es suficiente. De los sitios comprendidos en este estudio, sólo el RESA de Tecámac recuperaba el gas para su aprovechamiento.

Los modelos empleados en este estudio generan gráficos muy parecidos (con forma de campana, habitual en modelos de predicción de generación de biogás), debido a que ambos poseen el mismo fundamento. En la figura 2 se muestran cuatro proyecciones representativas de los tipos de SDF del presente estudio: 2a) RESA Bordo Poniente (clausurado), 2b) RESA Tlalnepantla, 2c) SC Isidro Fabela y 2d) SNC San Martín de las Pirámides. Con el MMB se obtienen en tres casos flujos mayores en el año de máxima producción (Fig. 2); por su parte, el modelo del IPCC predice en tres casos una mayor generación de biogás por más tiempo para cada sitio. Estas diferencias se deben principalmente a las tasas de generación potencial de $\mathrm{CH}_{4}\left(L_{0}\right)$ que emplean, siendo más altas las utilizadas por el $\mathrm{MMB}$, lo que lleva a considerar que la fracción orgánica de los residuos se agota en menor tiempo, además de que se ponderan automáticamente conforme avanza el tiempo de operación del SDF. Con el modelo del IPCC se proyecta una producción de biogás posterior a un periodo de aproximadamente 30 años después de que el SDF deja de recibir residuos, mientras que en el MMB la generación de biogás en la mayoría de los casos tiende a cero pasando ese periodo de tiempo. Lo anterior se puede observar en el cuadro IV, que muestra los flujos obtenidos con ambos modelos en ciertos años. De igual manera, las tasas de generación repercuten en los resultados obtenidos para el periodo 2017-2030, cuando se estimaba que los SDF estarían llegando al final de la vida útil. Por tal motivo, al dejar de depositarse residuos disminuirá gradualmente la emisión de metano. Realizar estimaciones con el modelo del IPCC es adecuado cuando se tienen los datos característicos del sitio, debido a que el cálculo es manual y no se realiza una proyección automática.

Al momento de analizar los resultados obtenidos mediante ambos modelos, es importante considerar que son simplemente estimaciones aproximadas del potencial de rendimiento de gas. Los modelos simplifican el complejo proceso de descomposición de los RSU, pero no toman en cuenta numerosos factores como el grado de descomposición aeróbica (antes de llegar al SDF), las limitaciones de nutrientes, la inhibición biológica, interacciones físicas y químicas y los requerimientos para la síntesis de biomasa.

Otro factor que los modelos empleados en este trabajo no toman en cuenta es la variación anual en la disposición de RSU, por lo que se empleó un dato promedio de disposición anual para cada SDF con una tasa de crecimiento del 1\% anual. De acuerdo con Gómez et al. (2009), los factores económicos y las actividades sociales determinan la cantidad y composición de los residuos generados en las diferentes estaciones y lugares, por lo que la generación de RSU varía a través del tiempo.

Por otro lado, en un estudio realizado durante un año en un SNC cerca de Morelia, Michoacán, González et al. (2011) reportaron que la producción 

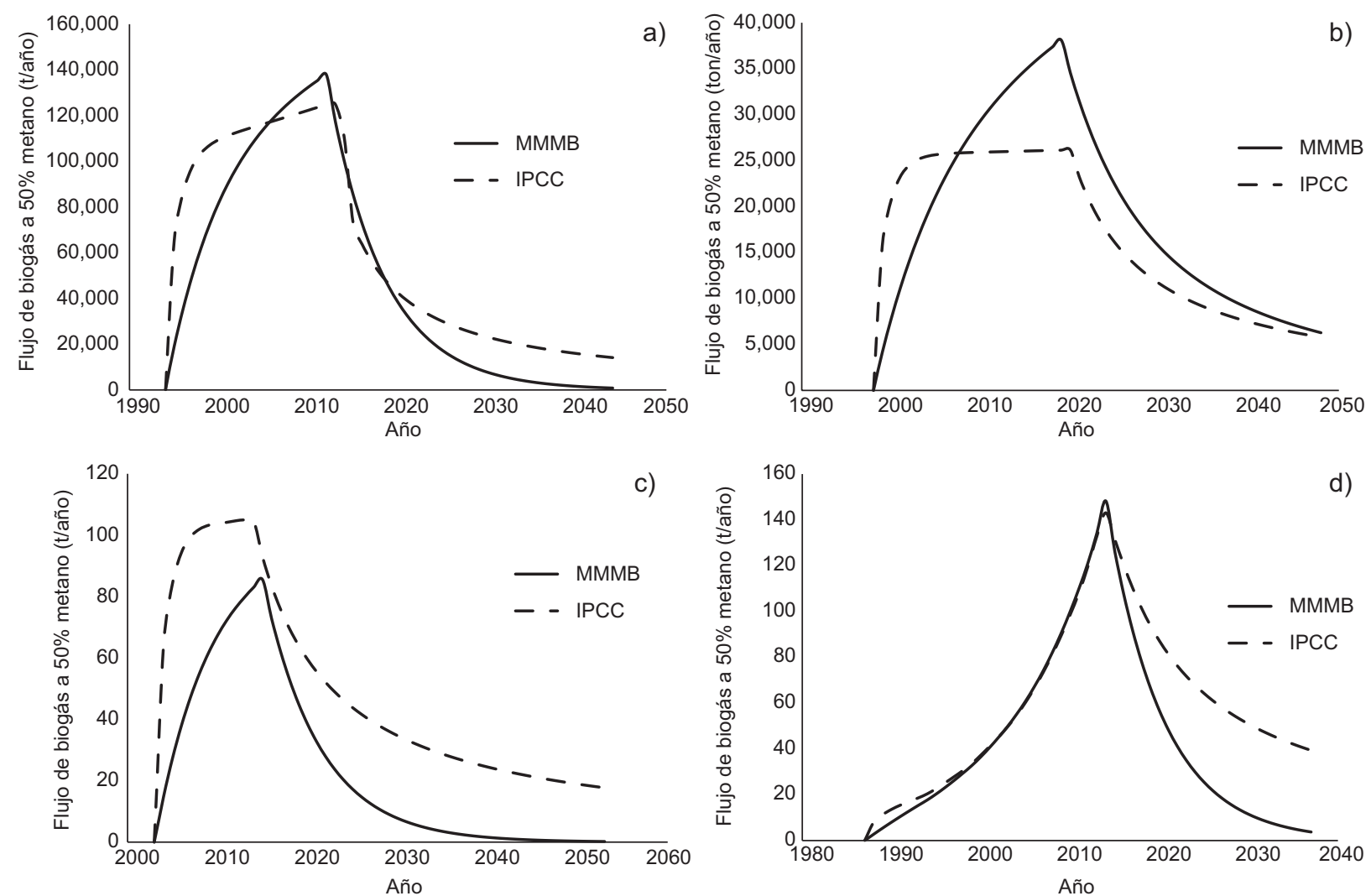

Fig. 2. Comparación de los resultados obtenidos por el Modelo Mexicano de Biogás y el modelo del Grupo Intergubernamental de Expertos sobre el Cambio Climático (IPCC) para los sitios de disposición final de a) Bordo poniente, b) Tlalnepantla, c) Isidro Fabela y d) San Martín de las Pirámides.

CUADRO IV. COMPARACIÓN DE LAS CANTIDADES DE GENERACIÓN DE BIOGÁS ESTIMADAS CON AMBOS MODELOS PARA LOS 30 SDF ESTUDIADOS.

\begin{tabular}{lrrrr}
\hline Año & \multicolumn{1}{c}{2017} & \multicolumn{1}{c}{2020} & \multicolumn{1}{c}{2030} & \multicolumn{1}{c}{2050} \\
\hline MMB (t/año) & 197730.85 & 192489.50 & 85335.34 & 11874.39 \\
IPCC (t/año) & 211209.20 & 223283.69 & 141153.07 & 69438.75 \\
Media & 204470.02 & 207886.60 & 113244.21 & 40656.57 \\
Diferencia (\%) & 6.82 & 16.00 & 65.41 & 484.78 \\
Desviación estándar & 8253.77 & 18857.52 & 34181.24 & 35250.83 \\
\hline
\end{tabular}

SDF: sitio de disposición final, MMB: Modelo Mexicano de Biogás, IPCC: Grupo Intergubernamental de Expertos sobre el Cambio Climático.

de biogás es mayor en temporada de lluvias en comparación con la época de estiaje; sin embargo, Colomer-Mendoza et al. (2013) no encontraron diferencias en la producción de biogás entre épocas de lluvias y estiaje en el RESA de Zapopan, Jalisco, a partir de los datos de generación durante dos años. Las discrepancias en el efecto de la variación estacional pudieran obedecer principalmente a que en un caso se trata de un RESA en operación y en el otro de un SNC clausurado, donde la cobertura podría ser inadecuada. Dado lo anterior, para obtener resultados más precisos es necesario realizar estudios de generación y composición a lo largo de un periodo de tiempo de cinco años, como mínimo, con el fin de observar la manera en que la emisión del gas se ve afectada por los comportamientos climáticos de cada SDF y 
realizar una estimación más sólida de esos sitios en México. Este tipo de trabajo ya se ha hecho en otros países, e.g., Klusman y Dick (2000) y Goldsmith et al. (2012), quienes afirman que el clima y el material de cobertura desempeñan un papel importante en las emisiones de metano.

En muchas ocasiones los datos son limitados (para muchos SDF son inexistentes) y difíciles de adquirir, dada la heterogeneidad e informalidad con la que operan muchos de los SDF, la falta de protocolos fiables en la toma de datos, la incertidumbre inherente a los métodos de recolección de gas empleados en diversos sitios, y los constantes cambios administrativos y operacionales que influyen en la disposición de los RSU y en el registro de la información. Por lo anterior, este estudio presenta una primera aproximación a la situación de las emisiones de una muestra de SDF en una zona de alta densidad poblacional.

Aguilar-Virgen et al. (2014) observaron que al cambiar los valores de $k$ y $L_{0}$ del MMB por mediciones realizadas en campo, obtuvieron flujos de biogás más altos que con el modelo; además, destacaron el mérito de obtener predicciones más precisas que ayudan a obtener una mejor proyección de generación de biogás en los SDF, si bien la generación total no se puede cuantificar.

\section{Validación parcial del MMB}

La recuperación estimada (RE) de biogás es sólo un porcentaje de la generación total estimada para los SDF y depende de la infraestructura que se tenga en cada sitio. Pocos son los RESA que cuentan con la tecnología para recuperar y cuantificar el flujo de biogás, debido a los costos de inversión que representan tanto la implementación como el mantenimiento de la infraestructura.

Los datos de generación mensual de biogás proporcionados por el personal del RESA de Tecámac para el periodo 2008-2013, se compararon con los datos estimados mediante el MMB; de esta manera se pudo observar que el volumen de biogás estimado por el MMB se aproxima al volumen reportado en campo
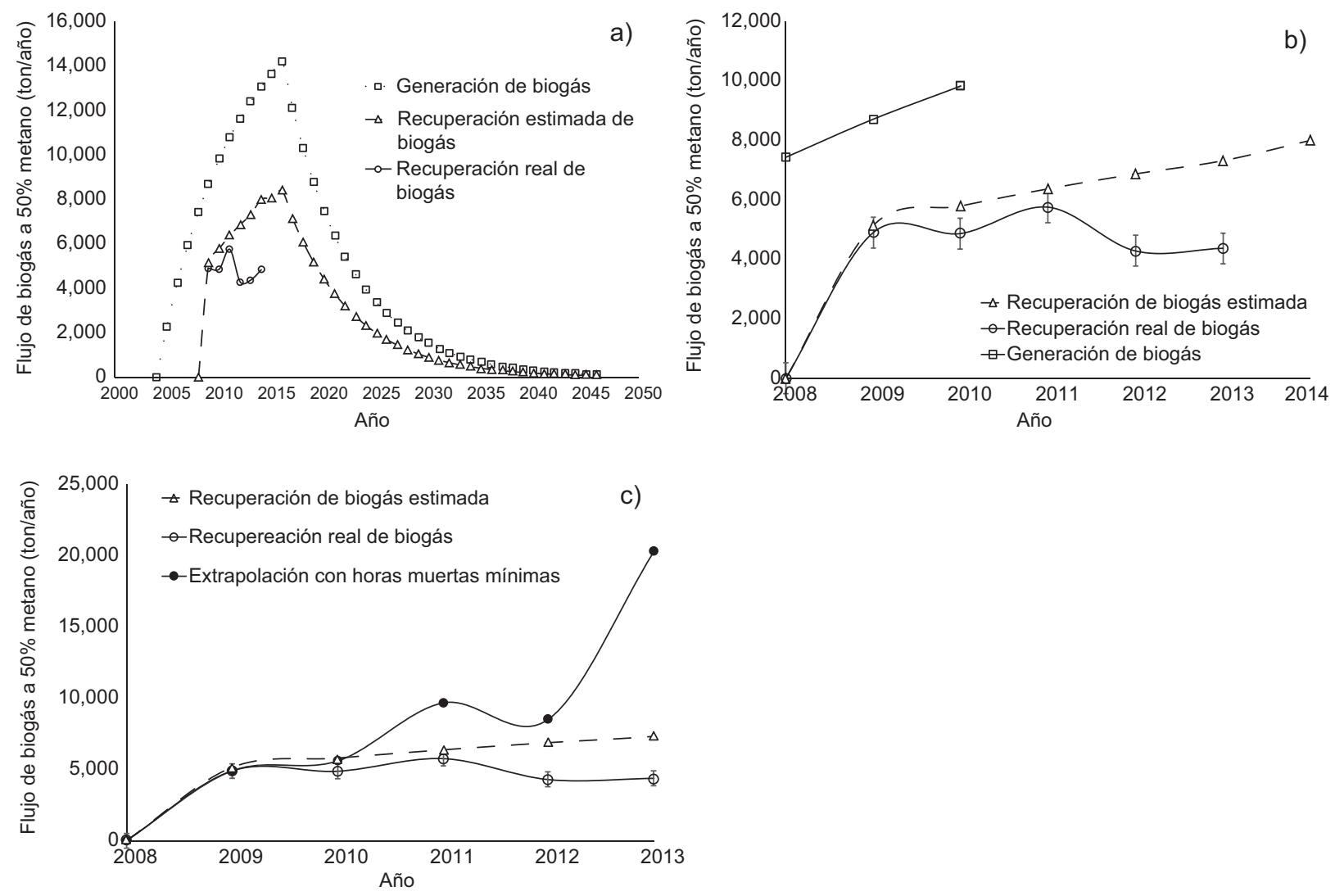

Fig. 3. Proyecciones de generación, recuperación estimada y recuperación real de biogás de Tecámac con el Modelo Mexicano de Biogás. (a) Gráfico completo de generación, recuperación estimada y recuperación real de biogás. (b) Acercamiento al promedio anual de 2008 a 2013. (c) Extrapolación de datos con el sitio de recuperación trabajando de manera ininterrumpida. 
(Fig. 3a). De acuerdo con el MMB y con el sistema de recolección (SR) de biogás de dicho sitio, se estimó una recuperación del $59 \%$ del biogás generado. Se aprecia que existen desviaciones entre la curva de RE y la recuperación real de biogás (detalle en Fig. 3b), sobre todo en los últimos años graficados, en los que el volumen de biogás recuperado disminuyó. Los datos reales reflejan los problemas operativos del $\mathrm{SR}$, ya que dicho sistema se interrumpió en varias ocasiones por problemas internos de la empresa y del proceso, mientras que en el MMB se considera que el SR opera en condiciones óptimas (toda la red en operación) y de manera ininterrumpida. No obstante, los datos del RESA se encuentran dentro de la desviación del MMB hasta 2012; posteriormente, las horas sin operación del SR se incrementaron de 740 en 2012 a 1725 en 2013.

Por otro lado, ya que el flujo de biogás se incrementa hasta el cierre de operaciones y se podría recuperar al menos el porcentaje que cubre el SR, se realizó una extrapolación de los datos que representarían la recuperación real de biogás, considerando el funcionamiento sin interrupciones en el SR, lo cual se observa en la figura 3c. Esto refleja que si el SR hubiera funcionado en condiciones óptimas, se habría capturado casi el triple del volumen de biogás en 2013 (4370.1 contra 11747.6 t/año de biogás).

\section{Contaminación ambiental por el biogás}

Por otro lado, en la figura 4 se muestra la estimación de la generación de biogás de los SDF estudiados, observándose que los municipios más cercanos a la CdMx son los que más biogás producen. Si bien es cierto que estos SDF son los mejor gestionados, no todos tienen un SR o de quema del biogás, e incluso teniéndolo hay una fracción del gas que se emite a la atmósfera y que por su cercanía con la zona más poblada del país representa un factor de contaminación y de riesgo importante para sus habitantes. Aunque el objeto de este trabajo es la estimación de $\mathrm{CH}_{4}$, hay otros contaminantes asociados con el mal manejo de los SDF, por ejemplo, los contaminantes criterio como $\mathrm{NO}_{x}, \mathrm{SO}_{\mathrm{x}}$ y $\mathrm{PM}_{10}$ y $\mathrm{PM}_{2.5}$, resultado de combustiones incompletas en los quemadores de los RESA o reacciones químicas del entorno.

Lo anterior se complica debido a que tanto en la CdMx como en la ZMVM se observa el efecto conocido como isla de calor, el cual consiste en que, en zonas urbanas, las superficies cubiertas por materiales como concreto y las grandes edificaciones obstruyen el libre flujo de aire y ocasionan que el calor de las construcciones se conserve por más tiempo durante las noches, en comparación con zonas rurales (Jáuregui-Ostos 2000). Así pues, el flujo del viento en la superficie es influenciado por el flujo del viento

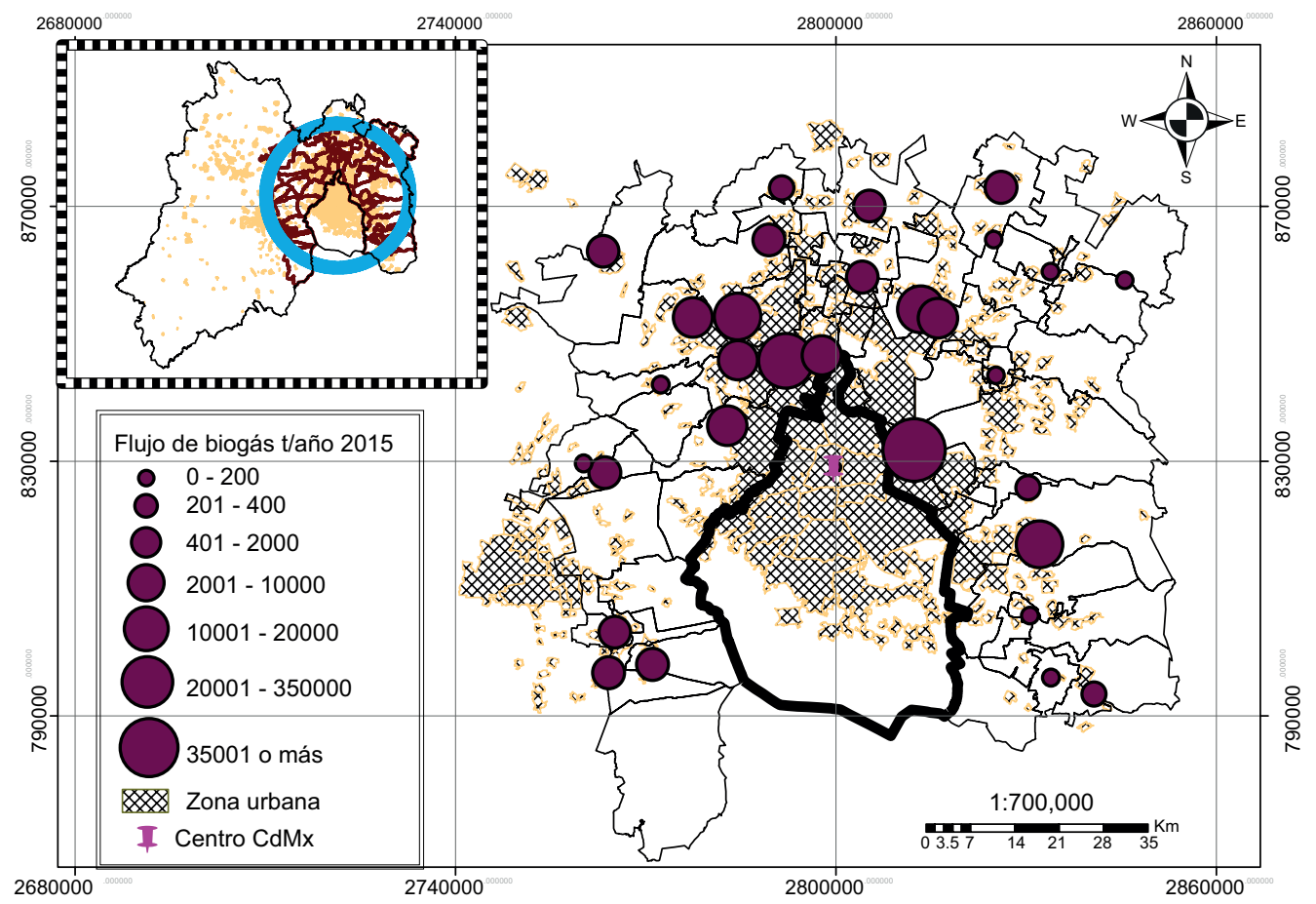

Fig. 4. Representación de la estimación del flujo de biogás generado en 2017. 
en niveles bajos y altos de la troposfera (Fig. 5). El efecto de las características orográficas (montañas circundantes) del Valle de México sobre el flujo del viento en los niveles bajos se deja sentir en los patrones de viento propios de las áreas montañosas que, de acuerdo con los cambios diurnos de temperatura, se torna ascendente durante el día (desde el valle hacia las montañas) y descendente por las noches (desde las montañsa hacia el valle). Asimismo, el viento en el Valle de México exhibe un flujo turbulento debido, nuevamente, a edificios, árboles, fuentes de calor, calentamiento de la superficie terrestre por el sol y otros obstáculos que presenta la zona urbana. Todos estos elementos favorecen trayectorias del viento convergentes, divergentes o vórtices (remolinos), tanto con giro ciclónico del viento (donde éste converge) como con giro anticiclónico (donde por lo general el viento diverge). Estos ejemplos se muestran en el informe climatológico ambiental del Valle de México de 2005 (SEDEMA 2005). El viento es sumamente variable de un día a otro, pero en todos los casos se repiten dos patrones: un flujo de norte a sur y otro de sur a norte, cuya confluencia da lugar a la formación de un vórtice ciclónico en la región centro de la CdMx. Finalmente, estos patrones provenientes del norte acarrean hacia la CdMx los contaminantes y partículas producidos en esa zona, lo cual, para efectos de este estudio, incluye una parte del biogás generado en los SDF del EM.

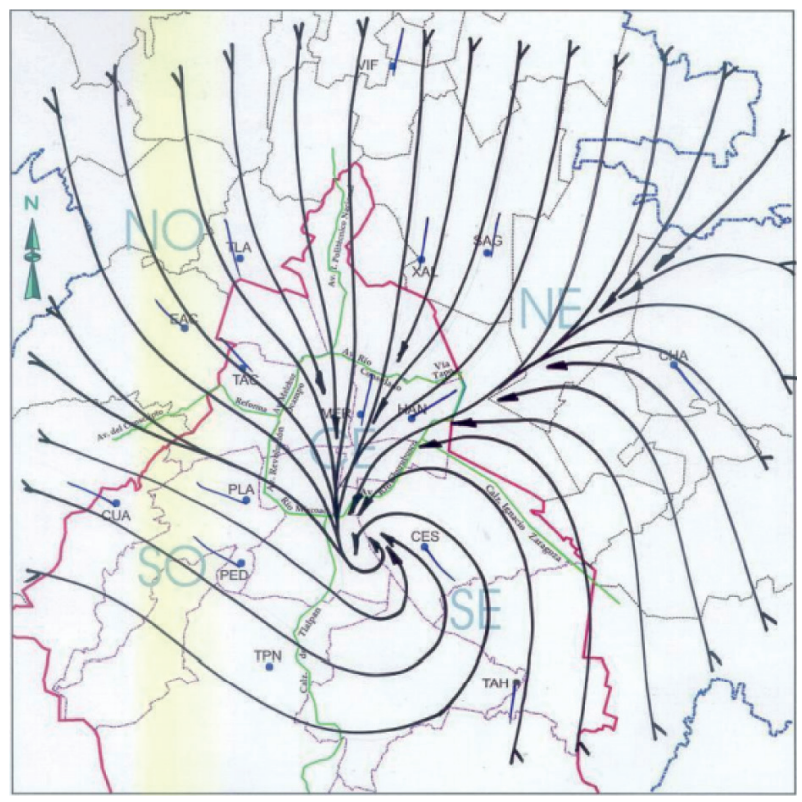

Fig. 5. Líneas de flujo de viento promedio anual en el Valle de México a las 18:00 horas (SEDEMA 2005).
En la CdMx se cuenta con un indicador que informa a la población sobre el estado de la calidad del aire: el índice de calidad del aire (ICA). Éste se calcula para cinco contaminantes criterio: dióxido de azufre, monóxido de carbono, dióxido de nitrógeno, ozono y partículas suspendidas.Cabe resaltar que el metano no está incluido en este índice. Según datos de la Comisión Ambiental de la Megalópolis (CAME 2016), durante el primer semestre de 2016 en 115 días se rebasaron los 100 puntos ICA; es decir, la calidad del aire estuvo fuera de la Norma de Salud Ambiental NOM-020-SSA1-2014 (SSA 2014), por lo que se implementó un programa extraordinario de contingencia ambiental atmosférica (6 de abril-30 de julio de 2016), el cual se enfocó a disminuir los contaminantes emitidos principalmente por el transporte (CaMe 2016). A pesar de todos estos esfuerzos, la mala calidad del aire en la CdMx y municipios contiguos del EM continuó durante y después de la vigencia del programa extraordinario, lo que lleva a pensar que existen otras fuentes de emisión de contaminantes que no se tomaron en cuenta para el plan de contingencia, como los gases generados por los SDF, ya que éstos, como se señaló anteriormente (Cuadro I), existen compuestos orgánicos volátiles o precursores de ozono. Lo anterior, aunado a la contribución del $\mathrm{CH}_{4}$ y los $\mathrm{NO}_{\mathrm{x}}$ al incremento del ozono troposférico (Ehhalt y Prather 2001), lo cual afecta el ICA.

\section{CONCLUSIONES}

A pesar de la falta de datos y de información sobre el manejo y operación de sitios de disposición final en el Estado de México, se logró estimar la generación de biogás en 30 sitios. La generación de biogás en un SDF es directamente proporcional al contenido de carbono orgánico biodegradable de los RSU y está en función del tipo de manejo con el cual son operados los SDF, así como de la velocidad de degradación de los residuos. Así pues, los sitios que generan la mayor cantidad de biogás también son los que se categorizan como RESA, que en este estudio resultaron ser la mitad de los sitios analizados. Por otro lado, los modelos empleados para la predicción de generación de biogás muestran una aproximación de lo que se puede llegar a producir en un SDF. Para que los modelos de predicción de efluentes de SDF lleguen a ser más precisos, se deberían tomar en cuenta numerosos factores que resulta difícil considerar, por la inestabilidad y numerosas variables del sistema. A pesar de que los modelos utilizados en este 
trabajo no consideran todas las variables presentes en los RESA, el pronóstico de impacto ambiental tiene relevancia, ya que la producción de biogás se lleva a cabo durante varias décadas y podrían tomarse medidas para evitar la emisión del gas y su migración, la cual empeora la calidad del aire de la ZMVM. Si bien los SDF más cercanos a la CdMx están mejor gestionados, aún no cuentan con SR o quema de gas eficientes. Además, la ubicación geográfica y urbanización que caracterizan a la CdMx propician la formación de flujos de aire en la zona centro que arrastran los contaminantes proveniente del norte, justo donde se ubican actualmente los SDF que emiten diversos compuestos gaseosos. A lo anterior hay que sumarle los contaminantes provenientes de SC y SNC. Finalmente, es necesario realizar mediciones que permitan relacionar las contingencias ambientales que se presentan en la ZMVM con las emisiones de contaminantes de SDF.

\section{REFERENCIAS}

Aguilar-Virgen Q., Taboada-González P., Ojeda-Benítez S. y Cruz-Sotelo S. (2014). Power generation with biogas from municipal solid waste: Prediction of gas generation with in situ parameters. Renew. Sust. Energ. Rev. 30, 412-419. https://doi.org/10.1016/j.rser.2013.10.014

CAME (2016). Informe de actividades correspondientes a la activación de los programas para contingencias ambientales atmosféricas en la zona metropolitana del Valle de México durante la temporada de ozono 2016. Comisión Ambiental de la Megalópolis, Ciudad de México, 59 pp.

Cárdenas-Moreno P.R. (2014). Estimación de biogás y lixiviados generados en sitios de disposición final de residuos sólidos urbanos del Estado de México. Tesis de Maestría. Unidad Profesional Interdisciplinaria de Biotecnología, Instituto Politécnico Nacional, Ciudad de México, México.

Cárdenas-Moreno P.R., Piña-Guzmán A.B., ColomerMendoza F.J., Carlos-Alberola M. y Robles-Martínez F. (2019). Evaluation of compliance with regulatory factors of waste disposal sites by using geographical information systems, case of study: State of Mexico. Rev. Int. Contam. Ambie. 35(4), 1025-1037. https:// doi.org/10.20937/RICA.2019.35.04.20

Chiemchaisri C. y Visvanathan C. (2008). Greenhouse gas emission potential of the municipal solid waste disposal sites in Thailand. J. Air Waste Manage. 58, 629-635. https://doi.org/10.3155/1047-3289.58.5.629

Colomer-Mendoza F.J., García-Darás F., Vives-Peris V. y Robles-Martínez F. (2013). Aspectos ambientales a considerar en vertederos: Emplazamiento y emisiones de biogás. DYNA 88, 726-730.

Colomer-Mendoza F.J., García-Darás F., EstebanAltabella J., Robles-Martínez F. y Aranda G. (2016). Emisiones gaseosas de un relleno sanitario en México. Comparación con los modelos de generación de biogás. Rev. Int. Contam. Ambie. 32, 113-122. https://doi. org/10.20937/RICA.2016.32.05.08

CONAPO (2019). Indicadores demográficos de la República Mexicana, en el año 2012. Consejo Nacional de Población, México [en línea]. http://www.conapo. gob.mx/work/models/CONAPO/Mapa_Ind_Dem18/ index_2.html 2/12/2019

Ehhalt D. y Prather M. (2001). Atmospheric chemistry and greenhouse gases. En: Climate Change 2001: The Scientific Basis. Contribution of Working Group I to the Third Assessment Report of the Intergovernmental Panel on Climate Change (Houghton J.T., Ding Y., Griggs D.J., Noguer M., van der Linden P.J., Dai X., Maskell K. y Johnson C.A. Eds.). Cambridge University Press, Cambridge, United Kingdom and New York, NY, USA, 239-288.

Friedrich E. y Trois C. (2011). Quantification of greenhouse gas emissions from waste management processes for municipalities - A comparative review focusing on Africa. Waste Manage. 31, 1585-1596. https://doi. org/10.1016/j.wasman.2011.02.028

Goldsmith C.D., Chanton J., Abichou, T., Swan N., Green R. y Hater G. (2012). Methane emissions from 20 landfills across the United States using vertical radial plume mapping. J. Air Waste Manag. Assoc. 62 (2), 183-197. https://doi.org/10.1080/10473289.2011.639480

Gómez G., Meneses M., Ballinas L. y Castells F. (2009). Seasonal characterization of municipal solid waste (MSW) in the city of Chihuahua, Mexico. Waste Manage. 29, 2018-2024. https://doi.org/10.1016/j. wasman.2009.02.006

González C., Buenrostro O., Márquez L., Hernández C., Moreno E. y Robles F. (2011). Effect of Solid Wastes Composition and Confinement Time on Methane Production in a Dump. J. Environ. Prot. 02, 1310-1316. https://doi.org/10.4236/jep.2011.210151

González-Cruz R. (2013). Diagnóstico de las condiciones actuales de operación de los sitios de disposición final del Estado de México. Tesis de Licenciatura. Unidad Profesional Interdisciplinaria de Biotecnología, Instituto Politécnico Nacional, Ciudad de México, México.

INECC (2018). Inventario nacional de emisiones de gases y compuestos de efecto invernadero 1990-2015. Instituto Nacional de Ecología y Cambio Climático, Ciudad de México, México, 851 pp.

INEGI (2015). Censo nacional de gobiernos municipales y delegacionales 2015. Módulo 6: residuos sólidos 
urbanos. Instituto Nacional de Estadística y Geografía [en línea]. https://www.inegi.org.mx/programas/ cngmd/2015/ 16/06/2020

INEGI (2019). Estadística por tema. Población total por entidad federativa. Instituto Nacional de Estadística y Geografía [en línea]. https://www.inegi.org.mx/app/ areasgeograficas/?ag=15 16/06/2020

Jáuregui-Ostos E. (2000). El clima de la Ciudad de México. 1a. ed. Plaza y Valdés, Ciudad de México, México, 131 pp.

Klusman R.W. y Dick C.J. (2000). Seasonal variability in $\mathrm{CH}_{4}$ emissions from a landfill in a cool, semiarid climate. J. Air Waste Manage. Assoc. 50 (9), 1632-1636. https://doi.org/10.1080/10473289.2000.10464201

Kumar S., Gaikwad S.A., Shekdar A.V., Shirsagar P.S. y Singh R.N. (2004). Estimation method for national methane emission from solid waste landfills. Atmos. Environ. 38, 3481-3487. https://doi.org/10.1016/j. atmosenv.2004.02.057

Lee U., Han J. y Wang M. (2017). Evaluation of landfill gas emissions from municipal solid waste landfills for the life-cycle analysis of waste-to-energy pathways. J. Clean. Prod. 166, 335-342. https://doi.org/10.1016/j. jclepro.2017.08.016

Machado S.L., Carvalho M.F., Gourc J.P., Vilar O.M. y Do Nascimento J.C.F. (2009). Methane generation in tropical landfills: Simplified methods and field results. Waste Manage. 29, 153-161. https://doi.org/10.1016/j. wasman.2008.02.017

Mou Z., Scheutz Ch. y Kjeldsen P. (2015). Evaluation and application of site-specific data to revise the first-order decay model for estimating landfill gas generation and emissions at Danish landfills. J. Air Waste Manage. Assoc. 65 (6), 686-698. https://doi.org/10.1080/1096 2247.2015.1008653

Ngnikam E., Tanawa E., Rousseaux P., Riedacker A. y Gourdon R. (2002). Evaluation of the potentialities to reduce greenhouse gases (GHG) emissions resulting from various treatments of municipal solid wastes (MSW) in moist tropical climates: Application to Yaounde. Waste Manage. Res. 20, 501-513. https:// doi.org/10.1177/0734242X0202000604

Pipatti R. y Svardal P. 2007. Eliminación de desechos sólidos. En: Directrices del IPCC de 2006 para los inventarios nacionales de gases de efecto invernadero. Vol. 5. Desechos (Eggleston H.S., Buendía L., Miwa K., Ngara T. y Tanabe K., Eds.). IGES, Japón.
SSA (2014). NOM-020-SSA1-2014. Salud ambiental. Valor límite permisible para la concentración de ozono $\left(\mathrm{O}_{3}\right)$ en el aire ambiente y criterios para su evaluación. Secretaría de Salud. Diario Oficial de la Federación, México, 19 de agosto.

SEDEMA (2005). Informe climatológico ambiental del Valle de México 2005. Secretaría de Medio ambiente del Gobierno del Distrito Federal. Ciudad de México, México.

SEDEMA (2015). Inventario de residuos solidos de la Ciudad de México, 2015. Secretaría de Medio ambiente del Gobierno de la Ciudad de México. Ciudad de México, México.

SEMARNAT (2003). NOM-083-SEMARNAT-2003. Especificaciones de protección ambiental para la selección del sitio, diseño, construcción, operación, monitoreo, clausura y obras complementarias de un sitio de disposición final de residuos sólidos urbanos y de manejo especial. Secretaría de Medio Ambiente y Recursos Naturales. Diario Oficial de la Federación, México, 20 de octubre.

SEMARNAT (2019). Residuos sólidos urbanos. Secretaría de Medio Ambiente y Recursos Naturales [en línea]. https://www.gob.mx/cms/uploads/attachment/ file/554385/DBGIR-15-mayo-2020.pdf

Themelis N.J. y Ulloa P.A. (2007). Methane generation in landfills. Renew. Energy 32 (7), 1243-1257. https:// doi.org/10.1016/j.renene.2006.04.020

Urrego-Martínez E. y Rodríguez-Miranda J.P. (2016). Aplicación de las metodologías EPA, mexicano e IPCC para la estimación de biogás, caso de estudio relleno sanitario Doña Juana, Bogotá, Colombia. Universidad y Salud 18(2), 338-344.

USEPA-SCS (2009). EP-W-06-023. Manual del usuario. Modelo Mexicano de Biogás v. 2.0. Manual. United States Environmental Protection Agency-SCS Engineers. Reston, EUA, $35 \mathrm{pp}$.

Zúñiga A. (2014). Comunicación personal del encargado del Relleno Sanitario de Tecámac. Proporcionó los datos del flujo de recuperación del biogás para el presente trabajo. 